\title{
A unilocular thymic cyst associated with true thymic hyperplasia: a challenging diagnosis especially in a child
}

This article was published in the following Dove Press journal:

International Medical Case Reports Journal

25 September 2015

Number of times this article has been viewed

\author{
Mona Mlika ${ }^{1,2}$ \\ Walid Gattoufi ${ }^{1,2}$ \\ Hazem Zribi \\ Emna Braham ${ }^{1,2}$ \\ Adel Marghli \\ Faouzi El Mezni ${ }^{1,2}$ \\ 'Department of Pathology, \\ Abderrahman Mami Hospital, Tunis, \\ Tunisia; ${ }^{2}$ Research Unit URI2SPI6, \\ Université Tunis El Manar, Faculté de \\ Médecine de Tunis, Tunis El Manar, \\ Tunisia
}

\begin{abstract}
We report a new case of a mediastinal mass in a 19-year-old patient corresponding microscopically to an association of unilocular thymic cyst and true thymic hyperplasia. Our aim is to highlight the absence of specificity of clinical and radiological findings and the necessity of a thorough sampling of the tumor in order to establish the diagnosis.
\end{abstract}

Keywords: true thymic hyperplasia, thymic cyst, microscopy, surgery

\section{Introduction}

Thymic cysts are uncommon mediastinal lesions accounting for 3\%-5\% of all masses in the mediastinum. ${ }^{1}$ They are located in the anterior compartment in all age groups and are usually discovered incidentally on chest X-ray. They have been reported with a variety of neoplastic, autoimmune, and infectious conditions. The coexistence of two unusual thymic lesions such as thymic cyst and true thymic hyperplasia (TTH) is an exceedingly rare condition. When reviewing the English literature, only three cases have been reported. ${ }^{2-4}$ TTH is a rare cause of anterior mediastinal mass in children. It is characterized by a thymic growth beyond the limits for the patient's age with a conservation of the thymic architecture. In this case, we present a challenging association of an acquired thymic cyst and TTH.

\section{Case report}

A 19-year-old man, without a particular past medical history, presented with a 2-week history of sudden left-sided chest pain associated with dyspnea and fever. The patient's consent was obtained for this case report. Our institute's Ethics Committee does not require approvals for this type of report. The physical examination was unremarkable. Pulmonary function, electrocardiogram, bronchoscopy, and coronary angiography did not reveal any abnormality. Posteroanterior and lateral chest radiograph showed a homogeneous left parahilar-sided mass of $6 \mathrm{~cm}$, which was well-demarcated. The chest computed tomography (CT) scan showed a large anterior mediastinal cystic mass lateralized to the left side frame evoking a cystic teratoma or a cystic thymoma without aggressive loco-regional signs (Figure 1). Tumor markers such as serum AFP and HCG levels were within normal levels. A mediastinoscopy was performed. Per-operatory examination showed a cystic mass located in the thymic compartment with extension to the contralateral side. The dissection was impossible because of multiple adhesions. Thus, a lateral thoracotomy was performed in order to completely resect the mass. Frozen section examination showed a fibrous cyst wall with many thymic lobules suggestive of thymoma.
Correspondence: Mona Mlika

Department of Pathology, Abderrahman Mami Hospital, Post Box 2036, Ariana,

Tunis 2037, Tunisia

Tel +21698538862

Fax +21671710780

Email mlika.zorgati.mona@hotmail.com 


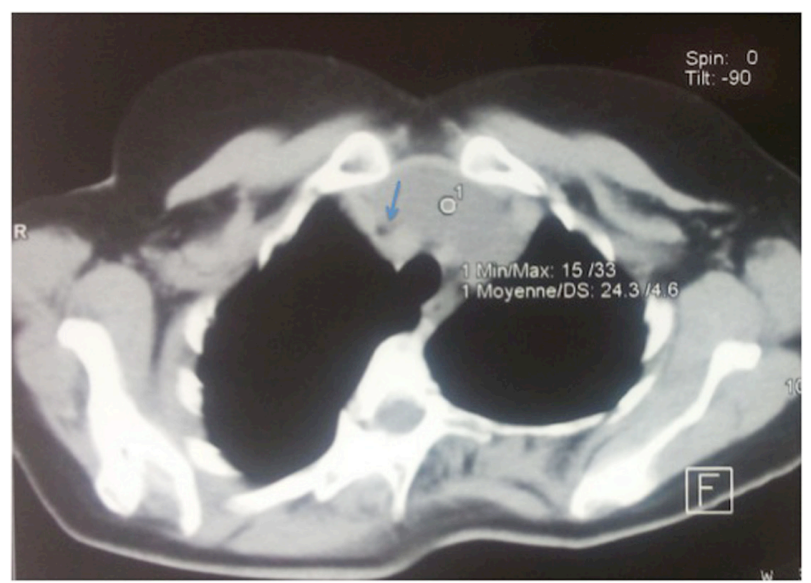

Figure I The chest computed tomography showed a large anterior mediastinal cystic mass lateralized to the left side frame evoking a cystic teratoma or a cystic thymoma without aggressive loco-regional signs (arrow).

A complete thymectomy with resection of the mediastinal fat was performed. Gross examination showed a cystic mass of $7 \mathrm{~cm}$, thin-walled with brownish liquid content (Figure 2). Microscopic examination showed a cyst wall lined by a flattened and unistratified epithelial lining overcoming thymic residues (Figure 3). Many Hassall's corpuscles - sometimes calcified - were observed. Thymic parenchyma seemed hyperplastic with conservation of the corticomedullary differentiation with the presence of many Hassall's corpuscles (Figure 3). An immunohistochemical study using the terminal deoxynucleotidyl transferase and cytokeratin antibodies was performed in order to rule out the diagnosis of thymoma. In fact, thymoma is characterized by the presence of two components: an epithelial one assessed by cytokeratin antibody and an immature inflammatory one highlighted by the deoxynucleotidyl antibody. We did not observe lymphoid

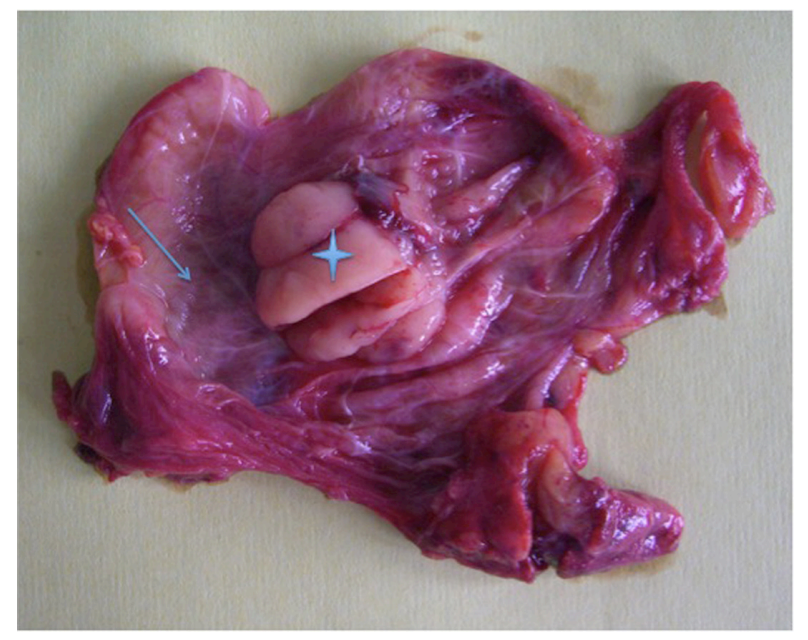

Figure 2 Gross examination showed a unilocular cystic mass (arrow) with some thymic lobules (star). follicles. Based on the microscopic findings, the diagnosis of cystic thymoma was initially suspected but rapidly ruled out because of the rarity of thymoma at that age. The diagnosis of a unilocular thymic cyst associated with TTH was established. The patient was discharged on the fourth postoperative day after an uneventful recovery.

\section{Discussion}

We reported this case in order to highlight the difficulty of establishing the microscopic diagnosis of TTH in association with thymic cysts. The major differential diagnosis is represented by cystic thymoma and differentiating both lesions may be challenging especially when radiological features are not specific. In this case, the diagnoses of thymoma or teratoma were suspected based on radiologic findings. Teratoma was easily ruled out based on microscopic findings. In fact, teratomas are characterized by a mixture of different tissues, that may be pancreatic, digestive, or respiratory. Ruling out a thymoma, especially a B1 thymoma, was more difficult. B1 thymomas are characterized by their organoid structure mimicking a normal thymus but they do not show many Hassall's corpuscles. ${ }^{5}$ Thymic cysts are rare lesions that account for $3 \%-5 \%$ of all mediastinal masses and represent approximately $1 \%-2 \%$ of anterior mediastinal tumors. ${ }^{1}$ These cysts are classified as unilocular or multilocular. Unilocular cysts, like our reported case, are considered to be congenital. ${ }^{6,7}$ On the other hand, multilocular thymic cysts (MTC) are believed to be acquired and were reported in association with teratoma, lymphoma, thymic carcinoma, and autoimmune disorders. ${ }^{6-10}$ Thymic cysts, including unilocular and multilocular cysts, are often discovered incidentally on routine chest radiograph and patients are most often asymptomatic. In a study performed by Suster and Rosai on 18 patients, seven patients presented with chest pain or discomfort. ${ }^{7}$ Our patient presented with chest pain, which may be caused by an acute hemorrhage within the cyst. Symptoms may be due to the enlargement of the cysts secondary to fluid accumulation. On chest CT scan, the lesion presents characteristically as a mediastinal mass containing multiple cysts in case of MTC or a unique cyst in case of unilocular cyst. ${ }^{11}$ Microscopically, a unilocular cyst is characterized by a unique cavity lined by flattened epithelial cells surrounded by a thymic parenchyma. In case of MTC, several cystic spaces are separated by thick walls containing dense lymphoid tissue. ${ }^{12}$ In our case, the microscopic features were challenging evoking a cystic thymoma because of the contiguity between the unilocular cystic lesion and thymic hyperplasia evoking a B1 thymoma. Analyses of 15 samples were performed in order to rule out this diagnostic. In fact, the multiplicity of the samples enabled to rule out many diagnostic features of thymomas including the lobulation of the proliferation 


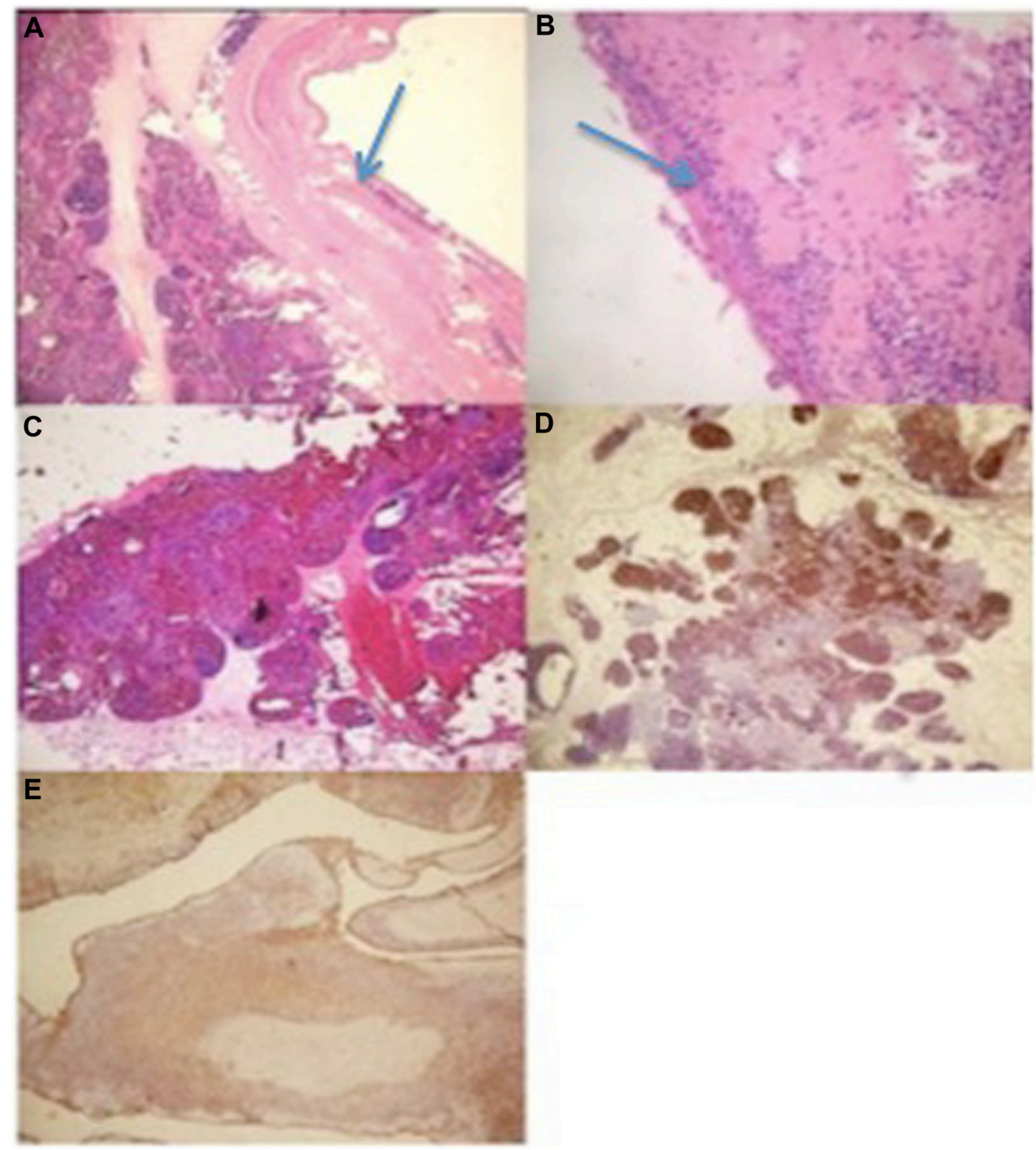

Figure 3 Microsopic findings.

Notes: (A) The coexistence of a cystic lesion (arrow) and true thymic hyperplasia (hematoxylin and eosin stain [HE] $\times 250$; (B) the epithelial lining (arrow) of the cyst was mainly flattened and focally hyperplastic $(\mathrm{HE} \times 400)$; (C) true thymic hyperplasia is characterized by the conservation of the thymic architecture which consists of a corticomedullary differentiation and the presence of many Hassall's corpuscles in the medulla $(\mathrm{HE} \times 250)$; (D) immunohistochemical study showing the positivity of the lymphocytes with the terminal deoxynucleotidyl transferase antibody $(\mathrm{HE} \times 250)$; (E) immunohistochemical study showing the positivity of the epithelial lining with the cytokeratin antibody $(\mathrm{HE} \times 250)$.

and the presence of two components, an epithelial one and an inflammatory one. In a thymic cyst, even if we can observe foci of thymic tissue, they remain organoid with a corticomedullary differentiation and many Hassall's corpuscles. Facing this association, other differential diagnoses may be evoked including nodular sclerotic Hodgkin disease, germ cell tumors, and mucosa-associated lymphoid tissue lymphoma. ${ }^{13,14}$ Consequently, thorough sampling of every thymic cyst must be carried out not only to establish an accurate diagnosis but also to exclude the possibility of neoplasm especially in a thickened cystic wall.

Treatment of thymic cysts is based on surgical excision, which must be as complete as possible. In a study of 18 cases reported by Suster and Rosai, two patients presented a recurrence of the cyst due to an incomplete surgical excision.
In our case, the unilocular thymic cyst was associated with TTH. TTH is seen in approximately $65 \%$ of patients with myasthenia gravis. ${ }^{14}$ Thymic cysts were considered as acquired in two cases and congenital in one case.

\section{Conclusion}

This report highlights the challenging diagnosis of an exceptional association of thymic cyst with TTH. The diagnosis is mainly based on the microscopic examination because of the nonspecific clinical and radiological characteristics.

\section{Disclosure}

The authors declare that they have no competing interests nor any personal or financial support. 


\section{References}

1. Davis JW, Florendo FT. Symptomatic mediastinal thymic cysts. Ann Thorac Surg. 1988;46(6):693-694.

2. Yatsuyanagi E, Kusajima K, Takezawa C. [A case of thymic cyst with thymic hyperplasia]. Nihon Kokyuki Gakkai Zasshi. 2003;41(5):336-340. Japanese.

3. Scharifker D. True thymic hyperplasia associated with a unilocular thymic cyst: an unusual combination not previously reported. Ann Diagn Pathol. 2006;10(1):32-35.

4. Eifinger F, Ernestus K, Benz-Bohm G, et al. True thymic hyperplasia associated with severe thymic cyst bleeding in a newborn: case report and review of the literature. Ann Diagn Pathol. 2007;11(5):358-362.

5. Weydert JA, De Young BR, Leslie KO; Association of Directors of Anatomic and Surgical Pathology. Recommendations for the reporting of surgically resected thymic epithelial tumors. Hum Pathol. 2009;40(7):918-923.

6. Kondo K, Miyoshi T, Sakiyama S, Shimosato Y, Monden Y. Multilocular thymic cyst associated with Sjögren's syndrome. Ann Thorac Surg. 2001;72(4):1367-1369.

7. Suster S, Rosai J. Multilocular thymic cyst: an acquired reactive process. Study of 18 cases. Am J Surg Pathol. 1991;15(4):388-398.
8. Suster S, Barbuto D, Carlson G, Rosai J. Multilocular thymic cysts with pseudoepitheliomatous hyperplasia. Hum Pathol. 1991;22(5): 455-460.

9. Moran CA, Suster S, El-Naggar A, Luna MA. Carcinomas arising in multilocular thymic cysts of the neck: a clinicopathological study of three cases. Histopathology. 2004;44(1):64-68.

10. Rakheja D, Weinberg AG. Multilocular thymic cyst associated with mature mediastinal teratoma: a report of 2 cases. Arch Pathol Lab Med. 2004;128(2):227-228.

11. Choi YW, McAdams HP, Jeon SC, et al. Idiopathic multilocular thymic cyst: CT features with clinical and histopathologic correlation. AJR Am J Roentgenol. 2001;177(4):881-885.

12. Nomori H, Horio $H$, Suemasu $K$, et al. A case of rapidly enlarging unilocular thymic cyst. J Clin Pathol. 2002;55(8):636-640.

13. Moran CA, Suster S. Mediastinal yolk sac tumors associated with prominent multilocular cystic changes of thymic epithelium: a clinicopathologic and immunohistochemical study of five cases. Mod Pathol. 1997;10(8):800-803.

14. Wekerle H. The thymus in myasthenia gravis. Ann N YAcad Sci. 1993;681:47-55.
International Medical Case Reports Journal

\section{Publish your work in this journal}

The International Medical Case Reports Journal is an international, peer-reviewed open-access journal publishing original case reports from all medical specialties. Previously unpublished medical posters are also accepted relating to any area of clinical or preclinical science. Submissions should not normally exceed 2,000 words or

\section{Dovepress}

4 published pages including figures, diagrams and references. The manuscript management system is completely online and includes a very quick and fair peer-review system, which is all easy to use. Visit $\mathrm{http}: / /$ www.dovepress.com/testimonials.php to read real quotes from published authors. 